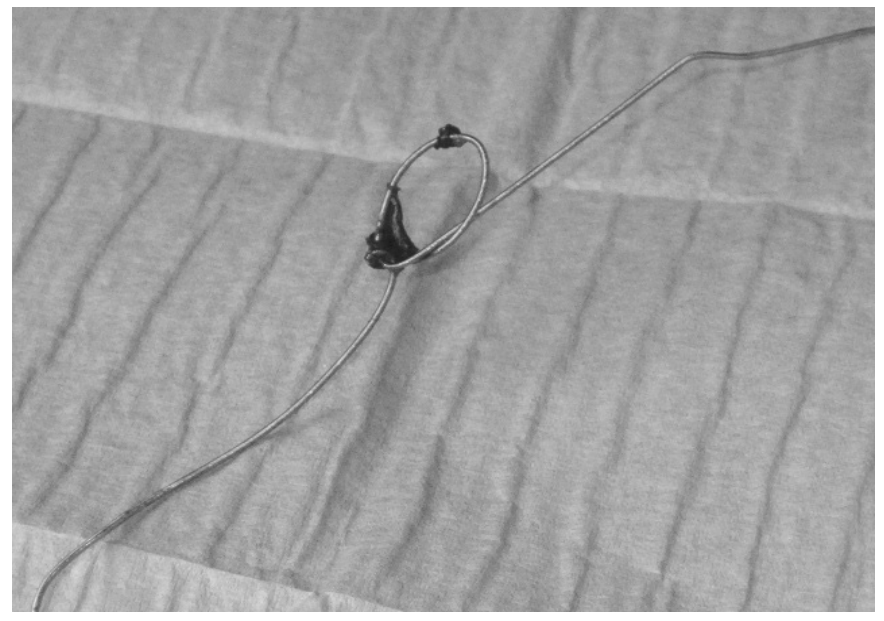

Fig. 1. Doble lazo con bucle en guía metálica de catéter venoso central.

\title{
Complicaciones infrecuentes en la cateterización de vías venosas centrales
}

\section{Sr. Director:}

Por todos son conocidas las posibles complicaciones de las vías venosas centrales, entre las que se incluyen las mecánicas y las infecciosas que desglosaremos más adelante.

Aportamos un caso con una peculiar imagen que nos deja dicho procedimiento:

Se trata de una paciente mujer de 91 años, con aceptable calidad de vida previa, y con antecedentes de diabetes mellitus insulín-dependiente con nefropatía secundaria, hipertensión arterial y obesidad, que ingresa en nuestro centro por cuadro de sepsis de origen urinario, y deterioro severo de la función renal junto con acidosis metabólica secundaria a lo previo, por lo que se decide cateterización de vía venosa central.

Durante la canalización de la vía venosa subclavia izquierda de la paciente no hubo complicaciones hasta que se intentó pasar el catéter a través de la guía metálica sin progresión del mismo. Se movilizó el cuello por la dificultad de progresión de la guía metálica, hasta que finalmente progresó parcialmente pero de forma insuficiente.

Sobra decir el apuro que presentó el médico que no podía extraer la guía, por lo que se realizó una pequeña incisión en la piel hasta que observó el pequeño lío, y tirando ligeramente pudo extraer lo que vemos en la imagen (Fig. 1).

Las complicaciones más frecuentes de la cateterización de vías venosas centrales se pueden clasificar en 2 grupos: 1. complicaciones mecánicas (1): a) malposición del extremo distal con posibilidad de producir arritmias, que son frecuentes pero habitualmente transitorias y no causan inestabilidad hemodinámica, perforación e incluso taponamiento, o bien ir en dirección distinta al trayecto de la vena canalizada. Por dichos motivos es recomendable siempre la realización de una radiografía de tórax, para comprobar su localización (2); b) embolia gaseosa, en relación con la entrada de aire durante la inserción o retirada del catéter; c) trombosis, relativamente frecuente aunque normalmente con escasa relevancia clínica, y desapareciendo tras la sustitución o retirada del catéter; puntualmente produce graves consecuencias ocasionando oclusión total permanente (3); d) punción arterial; e)

neumotórax, que en ocasiones llega a precisar de colocación de tubo de drenaje, para la evacuación del aire, dependiendo el porcentaje de aparición según la vía de acceso elegida (mayor en $\mathrm{V}$. subclavia) (4), y del grado de experiencia del médico; f) sangrado retroperitoneal en accesos de la V. femoral, que son potencialmente fatales por su dificultad diagnóstica; g) otras ("Pinch-off" síndrome (5), estenosis de vena subclavia, extravasación, laceraciones e incluso perforaciones de la pared venosa, sangrado prolongado habitualmente en personas con coagulopatías o trombocitopenias, pérdida de la guía metálica, etc.). 2. Complicaciones infecciosas (6), que son las más frecuentes y se pueden clasificar según el punto de inserción, metódica y el cuidado del catéter, que es con diferencia el factor más importante (7). Las complicaciones según la literatura varían de $1-40 \%$, debido a las diferentes formas de entender la definición de "infección de catéter" $(8,9)$. Los factores más frecuentes que pueden determinar el riesgo de infección son la enfermedad de base e infecciones intercurrentes, tipo de catéter y material, procedimiento y lugar de inserción, cuidado del catéter, etc.

Dadas las graves consecuencias que pueden tener estos procedimientos, cabe destacar nuevamente que uno de los factores principales en estas técnicas sería la experiencia por parte del médico, reduciéndose el número de complicaciones asociadas (10)

I. Marañés Antoñanzas, Y. López Gallardo, M. L. Abella Vázquez, A. García Pardo, M. Bethencourt Feria

Servicios de Medicina Interna y Medicina Familiar y Comunitaria. Hospital Nuestra Señora de La Candelaria. Santa Cruz de Tenerife

1. Polderman KH, Girbes ARJ. Central venous catheter use. Part 1: Mechanical complications. Intensive Care Med 2002; 28: 1-17.

2. Frasinelli P, Pasquale MD, Cipolle MD, Rhodes M. Utility of Chest Radiograph after guidewire exchanges of central venous catheter. Crit Care Med 1998; 26: 611-615.

3. Timsit JF, Farkas JC, Boyer JM. Central Vein Catheter- related thrombosis in Intensive Care patients incidence, risk factors and relationship with catheter- related sepsis. Chest 1998; 114: 207-213.

4. MacDonald S, Watt AJ, Mc Nally D. Comparison of technical success and outcome of tunnelled catheters inserted via yugular and subclavian approaches. J Vasc Interv Radiology 2000; 11: 225-231. 
5. Aitken DR, Minton JP. The "pinch-off sign": a warning of impending problems with permanent subclavian catheters. Am J Surg 1984; 148: 633-636.

6. Polderman KH, Girbes ARJ. Central venous catheter use. Part 2: Infectious complications. Intensive Care Med 2002; 28: $18-28$.

7. Egebo K, Toft P, Jacobsen CJ. Contamination of central venous catheter. The skin insertion wound is a major source of contamination. J Hosp Infect 1996; 32: 99-104.

8. Pittet D, Tarara D, Wenzel RP. Nosocomial bloodstream infection in critically ill patients: excess length of stay, extra costs and attributable mortality. JAMA 1994; 271: 15581560.

9. Reed CR, Sessler CN, Glauser FL. Central venous catheter infections: concepts and controversies. Intensive Care Med 1995; 21: 177-183.

10. Pearson ML. Guidelines for prevention of intravascular device - related infections: an overview. The hospital infection control practices committee. Am J Infect Control 1996; 24: 262-293. 